\title{
Study of the Phenomenon of Whistler Echoes
}

\author{
T. Laaspere, W. C. Johnson, and J. F. Walkup
}

\section{Contribution From the Radiophysics Laboratory, Thayer School of Engineering, Dartmouth College, Hanover, N.H.}

(Received July 6, 1964; revised November 5, 1964)

\begin{abstract}
In considering the propagation of long whistlers and whistler echo trains, the question arises about where the downcoming whistlers are reflected. The several suggestions that have been made include ground reflection and reflection at the lower boundary of the ionosphere. In either case, the echo of a daytime whistler would make several more passes through the absorbing $D$ region than the whistler itself, and we should expect whistlers occurring around noon to have a much smaller probability of having echoes than whistlers occurring at night. An analysis of several years of data obtained at the Dartmouth College whistler station yields the result, however, that although the average whistler rate is much higher at night than during the day, the probability of a whistler having an echo shows little change from midnight to midday. Consistent with this observation are the results of another study showing that the difference in the intensity of a noontime whistler and its echo may be only a few decibels.

If the theoretical predictions about absorption of whistler-mode waves are even nearly correct, our results on whistler echoes are incompatible with the lower-boundary or ground-reflection model. In no cases studied by us has the whistler echo been more intense than the whistler itself, and we do not at present favor the idea that whistler echoes are amplified in the magnetosphere. A model consistent with our results is one in which a large fraction of the energy of a downcoming whistler is reflected above $D$-region heights. In this model a whistler may be pictured as bouncing back and forth between the ionospheres of the two opposite hemispheres, with some of the energy "leaking through" to the ground at one or both ends of the path. Whistler observations could also be explained by a model in which the daytime transmission loss for VLF energy is high only for the first upward penetration of the ionosphere, but small once the energy is propagating in the "whistler mode."
\end{abstract}

\section{Introduction}

Whistlers are a type of audio-frequency electromagnetic waves of natural origin. Most whistlers are undoubtedly initiated by lightning flashes, but they are also known to be generated in other impulsive events, such as nuclear explosions. The frequency versus time behavior of whistlers is determined by the dispersive propagation of the initiating impulse along a propagation path following, more or less, lines of force of the earth's magnetic field. A "short whistler" is initiated by an impulse in the hemisphere opposite to that of the receiver and undergoes a one-way trip through the magnetosphere; a "long whistler" is initiated in the hemisphere of the receiver, where it is observed after it has been returned from the other hemisphere. The "echo" of a short whistler will thus have traveled through the magnetosphere three times, the echo of a long whistler four times. Often an echo has echoes of its own, resulting in a string of echoes of increasing dispersion-a whistler echo train. For observational results concerning the whistler phenomenon the reader is referred to the papers of Helliwell and Morgan [1959]; Helliwell and Carpenter [1961]; and Laaspere, Morgan, and Johnson [1963]. Whistler propagation theory has recently been summarized by Gallet [1963].
Eckersley [1928] appears to have been the first to give a description of whistler echo trains. They were described in more detail in Storey's [1953] classic paper in the context of the theory that whistlers travel from hemisphere to hemisphere along magnetic lines of force. The height of reflection of whistlers at the end of the path received little scrutiny by Storey, who spoke simply of reflection "from the earth's surface." A paper by Smith [1961], however, speaks explicitly of reflection "from the lower layers" (of the ionosphere), and Helliwell [1963] discusses in some detail the conditions which would lead to reflection of a downcoming whistler at the lower boundary of a model ionosphere. A nighttime rocket flight has recently shown that whistlers may indeed be present in the ionosphere, but not at the ground below [Cartwright, 1964].

The height of reflection of whistler-mode waves has also been considered by Helliwell, Katsufrakis, and Carpenter [1962] in their study of propagation of "whistler-mode" waves from Navy VLF stations. They suggested that rapid changes in the index of refraction, such as may be caused by a valley of ionization between the $E$ and the $F$ regions, could protect a round-trip signal from absorption in the $D$ region of the hemisphere opposite to the receiver. From the diurnal variation of the whistler-mode waves in both hemi- 
spheres they concluded, however, that at the frequencies involved the reflection probably takes place at the lower boundary of the ionosphere or from the ground.

Altman and Cory [1962] have recently predicted that even in the frequency range $3-5 \mathrm{kc} / \mathrm{s}$, where they find the minimum transmission loss for whistler-mode waves, absorption introduces a loss of about $15 \mathrm{~dB}$ in a one-way passage through the ionosphere during the day and about $2 \mathrm{~dB}$ at night. Altman and Cory's calculations are in general agreement with those of Leiphart, Zeek, Bearce, and Toth [1962], who arrived at an absorption loss of $27 \mathrm{~dB}$ for the day and $2 \mathrm{~dB}$ for the night for a wave of $18 \mathrm{kc} / \mathrm{s}$. (To the $18 \mathrm{kc} / \mathrm{s}$ figures just quoted, Hodara [1962] adds a reflection loss of $12 \mathrm{~dB}$ during the day and $10.5 \mathrm{~dB}$ at night.) Similar theoretical results on the transmission loss have been obtained by Swift [1962] and by Jesperson and Pitteway [1963] by a full-wave solution. Field intensity measurements in the ionosphere at $18 \mathrm{kc} / \mathrm{s}$ appear to support the high theoretical values of the transmission loss [Leiphart, Zeek, Bearce, and Toth, 1962; Lomax, 1961].

In the presence of the high transmission loss predicted by Altman and Cory and others, a daytime whistler echo would be an extremely unlikely event if reflection were at the ground or at the lower boundary of the $D$ region, since in both of these models the echo makes four more transits of the absorbing region than the whistler itself. From observations such as those to be discussed in sections 3 and 4 we know, however, that it is not particularly uncommon for a daytime whistler to have one or even several echoes. We will also show that the intensity decrement between a daytime whistler and its echo is sometimes only a few decibels. We conclude that either the daytime ionospheric transmission loss of whistlers is much lower than the theories predict, or the models involving whistler reflection at the ground or at the lower boundary of the ionosphere are inapplicable at least during the observing periods around noon when the $D$-region absorption reaches its maximum value.

\section{Models of Whistler Propagation}

We suggest that the low values of intensity decrements in a daytime whistler echo train can be explained by "ionospheric trapping", which is based on the idea of reflection of the downcoming wave in the ionosphere. In this model a whistler may be pictured bouncing back and forth between the ionospheres of the two hemispheres, with reflections occurring above the region of high absorption. Both the gound-level whistler and its echo represent in this model only a "leakage signal" from above, and since both leakage signals pass through the region of highest absorption the same number of times (once), the intensity decrement between a whistler and its echo could be quite small.

A schematic representation of the ionospheric trapping model in its simplest form is compared with the ground reflection model in figure 1 , where region 1 represents the ground in the hemisphere of the whistler source and region 5 the ground in the other hemi-

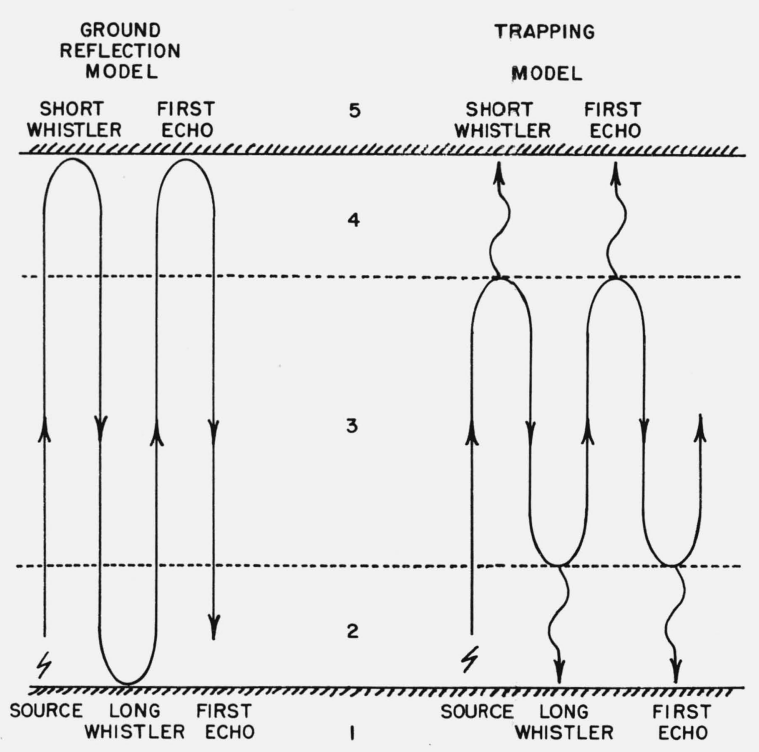

FigURE 1. Two models of whistler propagation.

sphere. In figure 1 the space between the two ground surfaces is divided only into three regions by two partially reflecting layers, interfaces, or other gradients existing in the ionosphere: regions 2 and 4 should be considered to represent the lower ionospheres of the two hemispheres, region 3 the upper ionosphere and the magnetosphere. It is probable that the actual situation is best represented by a trapping model involving more than two reflecting regions. Our considerations could easily be extended to the more general case, but this has not been done in this paper to keep the arguments as simple as possible.

The intensity decrements between component waves in a whistler echo train are derived in the appendix for several different propagation models. As shown by (1), in the ground reflection model the intensity decrements are determined by reflection losses suffered by a component wave at all of the interfaces of figure 1 and by absorption losses in passing twice through each of the regions 2,3 , and 4 . If the whistler echo train is trapped in region 3 , however, then, as is shown by (5), the intensity decrements are independent of absorption in regions 2 and 4, being determined only by absorption in region 3 and by the reflection coefficients of the interfaces $3-2$ and 3-4. The intensity decrements for mixed models, in which one of the ionospheric interfaces of figure 1 is removed, are given by expressions (6) and (7). If absorption in the lower ionosphere is the controlling factor, the intensity decrements in a mixed model lie between those of the ground reflection model (large decrements) and the ionospheric trapping model (small decrements).

It is important to note that a situation resulting in small intensity decrements in a whistler echo train may be far from optimum from the point of view of observing whistlers or whistler echo trains. For example, (5) predicts the smallest intensity decrements 
for perfect ionospheric trapping, but it is clear that in that case the whistler echo train would not be observed at a ground station. (It would, however, be observed by a satellite-borne receiver.) It is also clear that the propagation conditions of short whistlers should be best in the ground reflection model since the introduction of a partially reflecting layer in either hemisphere adds additional (reflection) losses in the path of the short whistler. The observing conditions of a long whistler may, on the other hand, be either degraded or improved depending on whether the layer is introduced in the hemisphere of the observer or in the opposite hemisphere.

Table 1 contains a summary of how partially reflecting layers affect the observing conditions of whistlers and whistler echoes if the lower-ionosphere absorption is high. Of course, if absorption in the lower ionosphere were negligible, partially reflecting layers would in general only degrade the propagation conditions.

TABLE 1. Effect of partially reflecting layers in the middle and upper ionosphere on the observing conditions of whistlers and whistler echoes if absorption in the lower ionosphere is high in both hemispheres.

\begin{tabular}{|c|c|c|c|}
\hline \multirow[b]{2}{*}{$\begin{array}{l}\text { Location of the partially reflect- } \\
\text { ing layers }\end{array}$} & \multicolumn{3}{|c|}{ Observing conditions for } \\
\hline & $\begin{array}{l}\text { Short } \\
\text { whistlers }\end{array}$ & $\begin{array}{c}\text { Long } \\
\text { whistlers }\end{array}$ & $\begin{array}{l}\text { Echo } \\
\text { trains }\end{array}$ \\
\hline Neither hemisphere.... & Best & Poor & Worst \\
\hline Other hemisphere........ & & Best & $\begin{array}{l}\text { Inter- } \\
\text { mediate }\end{array}$ \\
\hline Observer's hemisphere... & $\begin{array}{l}\text { Inter- } \\
\text { mediate }\end{array}$ & Worst & $\begin{array}{l}\text { Inter- } \\
\text { mediate }\end{array}$ \\
\hline Both hemispheres... & Worst & $\begin{array}{l}\text { Second } \\
\text { best }\end{array}$ & Best \\
\hline
\end{tabular}

\section{Study of Echo Statistics}

If there is, in fact, such a large difference between the daytime and the nighttime values of the ionospheric transmission loss for whistler-mode waves as has been predicted and reported, and if echo trains are produced by passing through the absorbing $D$ region in both hemispheres, we would expect the nighttime whistlers to have a much larger probability of having echoes than daytime whistlers, if the latter have any echoes at all.

The absence of any such pronounced diurnal variation in echo statistics at our local station ( $\mathrm{N} 55.2^{\circ}$, W $4.2^{\circ}$ geomagnetic; $\mathrm{N} 43.7^{\circ}, \mathrm{W} 72.3^{\circ}$ geographic) was first reported by Morgan and Johnson [1960]. We have now looked at the echo statistics in more detail. The results of the new studies are given in figures 2 and 3.

In figure 2 we have plotted the percentage of daytime and nighttime whistlers having one or more echoes (1959 June-1963 Apr.). In the hope of making the difference between the day and nighttime probabilities of echo occurrence more striking, daytime results were calculated using the 2-min hourly observing periods around local noon (1050-1450 hr local time), whereas the nighttime results were computed using the periods around local midnight $(2250-0150 \mathrm{hr}$ ). The data were grouped into different seasons as follows: Winter - December, January, February; Spring March, April; Summer-June, July; Fall-September, October.

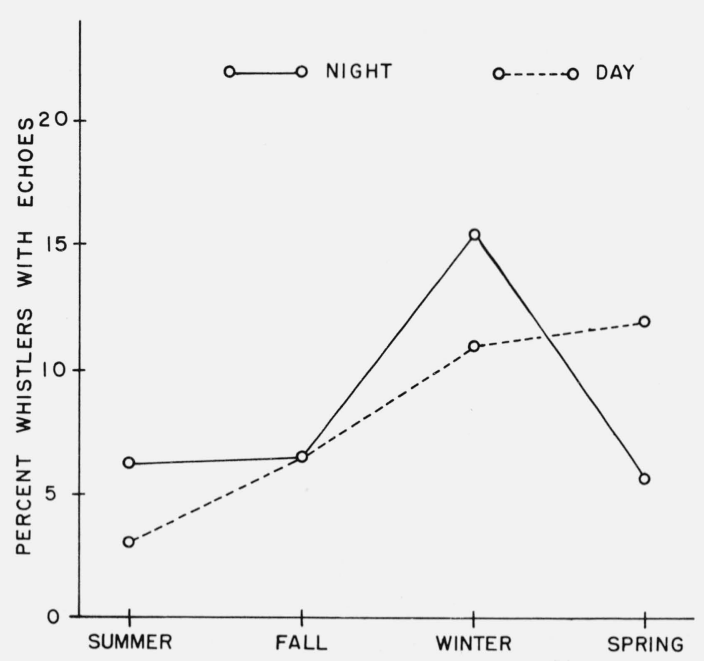

Figure 2. Percentage of whistlers having echoes in the observing periods near midday and midnight (1959 June-1963 Apr.).

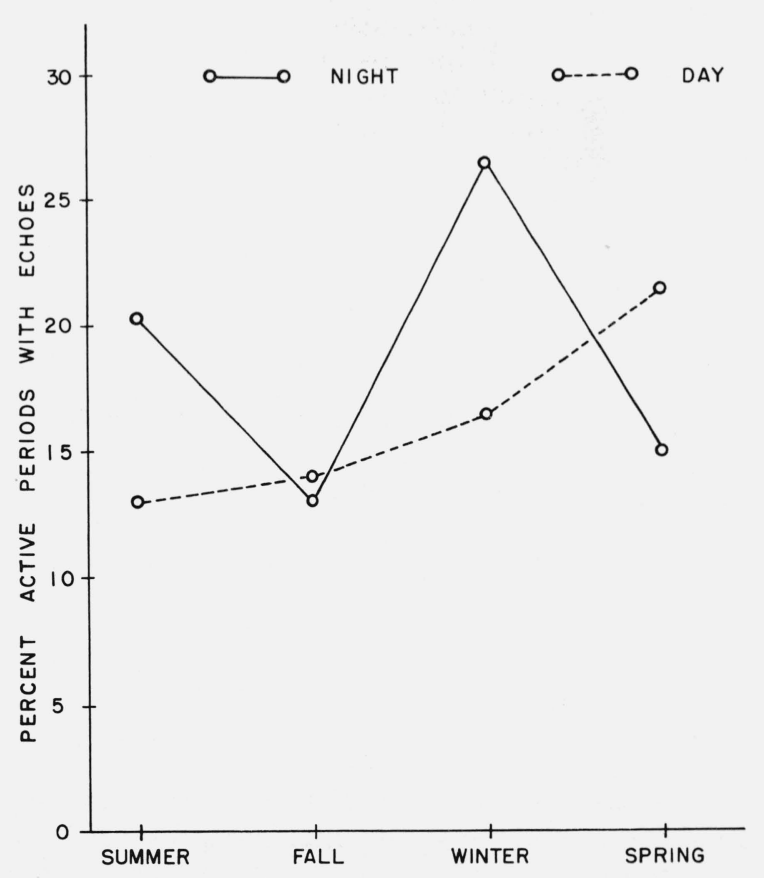

FIGURE 3. Percentage of whistler-active observing periods having echoes in the observing periods near midday and midnight (1957 Oct-1964 Mar.). 
Similar information for a longer period of time (1957 Oct.-1964 Mar.) is presented in figure 3, where we have plotted the percentage of "whistler-active" observing periods in which at least one whistler had one or more echoes. This is relatively easy to do, whereas the determination of the actual percentage of whistlers having echoes becomes unreliable if aurally monitored data are used and the whistler rate exceeds about 25 per minute. Actually, insofar as a comparison of day and night echo behavior in a given season is concerned, even the ordinate of figure 3 can be considered to be proportional to the probability of a whistler having an echo. This is because for a fixed whistler rate the percentage of active observing periods containing at least one echo should be proportional to the percentage of whistlers having echoes. For the data used in figure 3 the whistler rates at noon and at midnight in active periods were in fact surprisingly close: 4.4 versus 3.3 per minute in summer, respectively, 2.7 versus 2.9 in fall, 1.6 versus 1.8 in winter, and 1.6 versus 2.1 in spring.

Figures 2 and 3 show a surprisingly small difference in the probability of daytime and nighttime whistlers having echoes. While this is not what we would expect if the whistler echo train passed through a highly absorbing daytime $D$ region, the result does not necessarily mean that the intensity decrements in whistler echo trains are the same at midday and midnight, since in addition to the intensity decrement, the likelihood of observing an echo depends also on the intensity of the whistler and on the effective receiver noise level. At audio frequencies the latter is practically always determined by the level of atmospheric noise, which at our local station shows a diurnal maximum at night and minimum in the forenoon. Seasonally the noise is highest in local summer and lowest in winter. The diurnal variation of atmospheric noise thus favors the detection of echoes at midday, which may explain at least in part why the probability of a whistler having an echo may actually be higher at noon than at midnight, as in local spring. The seasonal variation of atmospheric noise may explain the winter peak in figures 2 and 3 . The seasonal variation of the probability may, however, also be caused by some effect associated with the high asymmetry of the whistler path with respect to the geographic equator-the area magnetically conjugate to our local station lies off the coast of Antarctica in the Bellinghausen Sea area. (For more information on our whistler station see Laaspere, Morgan, and Johnson [1963].)

\section{Study of Individual Echo Trains}

It would obviously be very difficult to deduce any estimates for the intensity decrements in whistler echo trains from the statistical analysis presented in the previous section. For this reason it was decided to actually measure the decrements in a number of whistler echo trains received at our local station.

Only a limited number of the whistler echo trains that have been recorded on magnetic tape were found suitable for this study, mainly because of the interference caused by atmospheric noise. The echo trains selected were passed through a voltage limiter (to discriminate against impulsive atmospherics), bandpass filter, rectifier, and amplifier. The output of the amplifier was recorded on paper and the output intensities determined by measuring the areas under the time versus output traces. The filter was designed to pass the band of frequencies from 3.3 to $4.7 \mathrm{kc} / \mathrm{s}$, which is approximately the band containing most of the energy of many of the echo trains observed by us. This is in agreement with Storey's [1953] observation that the "low intensity decrement is found only over a band about $1 \mathrm{kc} / \mathrm{s}$ wide centered on a frequency between 3 and $4 \mathrm{kc} / \mathrm{s}$." Note also that this is approximately the frequency band in which Altman and Cory [1962] predict the minimum transmission loss. It is interesting to note, however, that for propagation below the ionosphere this is just the frequency range of a pronounced absorption band [Watt and Maxwell, 1957].

The results of the study are presented in tables 2 and 3 , where it can be seen that for the echo trains analyzed, not only the night but also the daytime decrements are all less than $7 \mathrm{~dB}$. The daytime values are clearly much smaller than predicted by the theoretical studies mentioned previously.

TABLE 2. Decrease in intensity of successive components in a number of Dartmouth nighttime whistler echo trains in the band 3.3$4.7 \mathrm{kc} / \mathrm{s}$.

\begin{tabular}{|c|c|c|c|c|c|c|c|}
\hline & \multirow{2}{*}{ Time of occurrence } & \multicolumn{2}{|c|}{$\begin{array}{l}\text { Whistler and } \\
\text { first echo }\end{array}$} & \multicolumn{2}{|c|}{$\begin{array}{l}\text { First and } \\
\text { second echo }\end{array}$} & \multicolumn{2}{|c|}{$\begin{array}{l}\text { Second and } \\
\text { third echo }\end{array}$} \\
\hline & & $\begin{array}{c}\text { Power } \\
\text { ratio }\end{array}$ & $\mathrm{dB}$ & $\begin{array}{l}\text { Power } \\
\text { ratio }\end{array}$ & $\mathrm{dB}$ & $\begin{array}{c}\text { Power } \\
\text { ratio }\end{array}$ & $\mathrm{dB}$ \\
\hline $04 \mathrm{Ma}$ & . $196022: 35: 23$ EST & 2.3 & 3.6 & 1.9 & 2.8 & & \\
\hline $04 \mathrm{Ma}$ & $196023: 35: 53$ EST & 2.5 & 4.0 & & & & \\
\hline $18 \mathrm{De}$ & $196023: 50: 19$ EST & 1.8 & 2.5 & & & & \\
\hline $18 \mathrm{Dec}$ & $196023: 50: 46$ EST & 1.4 & 1.5 & 1.7 & 2.3 & 1.9 & 2.8 \\
\hline $19 \mathrm{Dec}$ & $196000: 50: 03$ EST & 1.5 & 1.8 & 1.9 & 2.8 & & \\
\hline $19 \mathrm{Dec}$ & $196000: 51: 24$ EST & 1.5 & 1.8 & 1.6 & 2.0 & & \\
\hline $19 \mathrm{Dec}$ & $196001: 50: 03 \mathrm{EST}$ & 2.7 & 4.3 & & & & \\
\hline $19 \mathrm{Dec}$ & $196001: 50: 24$ EST & 2.5 & 4.0 & & & & \\
\hline $19 \mathrm{Dec}$ & $196001: 51: 10$ EST & 3.5 & 5.4 & & & & \\
\hline $19 \mathrm{Dec}$ & $196001: 51: 25$ EST & 2.3 & 3.6 & & & & \\
\hline $19 \mathrm{De}$ & $196001: 51: 35$ EST & 4.4 & 6.4 & & & & \\
\hline $05 \mathrm{Fel}$ & $196122: 50: 24$ EST & 1.9 & 2.8 & & & & \\
\hline $19 \mathrm{Fel}$ & $196100: 50: 00$ EST & 2.6 & 4.1 & 1.7 & 2.3 & & ... \\
\hline $19 \mathrm{Fel}$ & $196100: 51: 00$ EST & 1.6 & 2.0 & & & & \\
\hline
\end{tabular}

TABLE 3. Decrease in intensity of successive components in a number of Dartmouth daytime whistler echo trains in the band 3.3$4.7 \mathrm{kc} / \mathrm{s}$.

\begin{tabular}{|c|c|c|c|c|}
\hline \multirow{2}{*}{ Time of occurrence } & \multicolumn{2}{|c|}{$\begin{array}{l}\text { Whistler and } \\
\text { first echo }\end{array}$} & \multicolumn{2}{|c|}{$\begin{array}{c}\text { First and } \\
\text { second echo }\end{array}$} \\
\hline & $\begin{array}{l}\text { Power } \\
\text { ratio }\end{array}$ & $\mathrm{dB}$ & $\begin{array}{c}\text { Power } \\
\text { ratio }\end{array}$ & $\mathrm{dB}$ \\
\hline 26 Oct $195915: 36: 03$ EST & 2.6 & 4.1 & 2.6 & 4.1 \\
\hline 04 Jun $1960 \quad 10: 35: 48$ EST & 2.2 & 3.4 & & \\
\hline 04 Jun $1960 \quad 10: 36: 40$ EST & 2.3 & 3.6 & & \\
\hline 04 Jun $196012: 35: 44$ EST & 4.2 & 6.2 & & \\
\hline 19 Dec $196013: 51: 26$ EST & 2.8 & 4.5 & 2.6 & 4.1 \\
\hline 16 May $1961 \quad 13: 50: 40$ EST & 4.6 & 6.6 & & \\
\hline
\end{tabular}


It could perhaps be argued that the small intensity decrements might be the result of a mechanism such as that suggested by Storey [1953]. He reports having observed whistler echo trains in which the first few echos appeared to increase in amplitude, stating that "this occurrence might be caused by successive whistlers (i.e., echoes) being focused at slightly different points on the earth's surface, the natural decay of the amplitude of the first few whistlers being overridden by the approach of the point of focus towards the observer." Extensive synoptic observations of whistlers conducted subsequent to Storey's work at the "Whistlers-East" network of Dartmouth Collegeand the "Whistlers-West" network of Stanford University have shown, however, that such a "creep effect" is not important. In aural monitoring of thousands of whistler echo trains received at our local whistler station over the past $6 \mathrm{yr}$, we have not discovered a single clear case of a whistler echo being more intense than the whistler itself. Note also (tables 2 and 3) that in the cases where it was possible to determine the intensity decrement of more than one echo, the decrement remained relatively constant, which is good evidence against the "creep" idea.

It might also be suggested that the results are consistent with the ground reflection model if some amplification of the whistler echo trains has taken place in the magnetosphere, but the arguments just given against the "creep" idea oppose the amplification idea with equal validity: It is hard to picture an amplification mechanism which so often falls short by only a few decibels from bringing the intensity of the echo up to that of the whistler itself without ever overcompensating for the path loss. Also, if an echo is subject to a larger transmission loss during the day than at night, the amplification mechanism would have to be more effective during the day than at night to explain our echo results.

\section{Conjugate-Area Studies}

\subsection{The Situation During Dartmouth's "Echo-Active" and "Whistler-Active" Periods}

In an attempt to elucidate the conditions leading to the occurrence of whistler echo trains, we have compared the whistler rates at Dartmouth and at the southern-hemisphere stations of Ellsworth and Port Lockroy for two different sets of observing periods: those in which both whistlers and echoes were observed at Dartmouth (Dartmouth's "echo-active" periods) and those in which only whistlers were observed (Dartmouth's "whistler-active" periods).

Figure 4 gives the results of the study for the northern-hemisphere summer months for observing periods near midnight, in the morning, and at midday. The results indicate that whistler rates in the southern hemisphere are practically identical in the two sets of observing periods. On the other hand, at Dartmouth the rates in the echo-active periods are considerably higher than in the whistler-active periods.

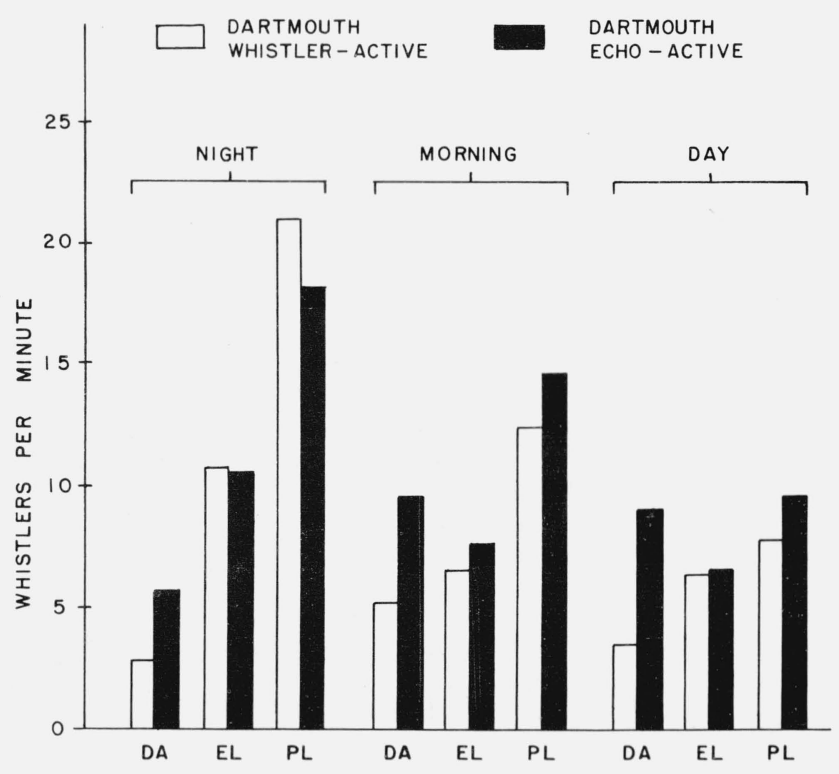

Figure 4. The whistler rates at Dartmouth (DA), Ellsworth (EL), and Port Lockroy (PL) at night (0335-0635 UT), in the morning (1035-1235 UT), and at midday (1535-1935 UT) in the months of June and July, 1958-1961, during those observing periods which were whistler-active and echo-active at Dartmouth.

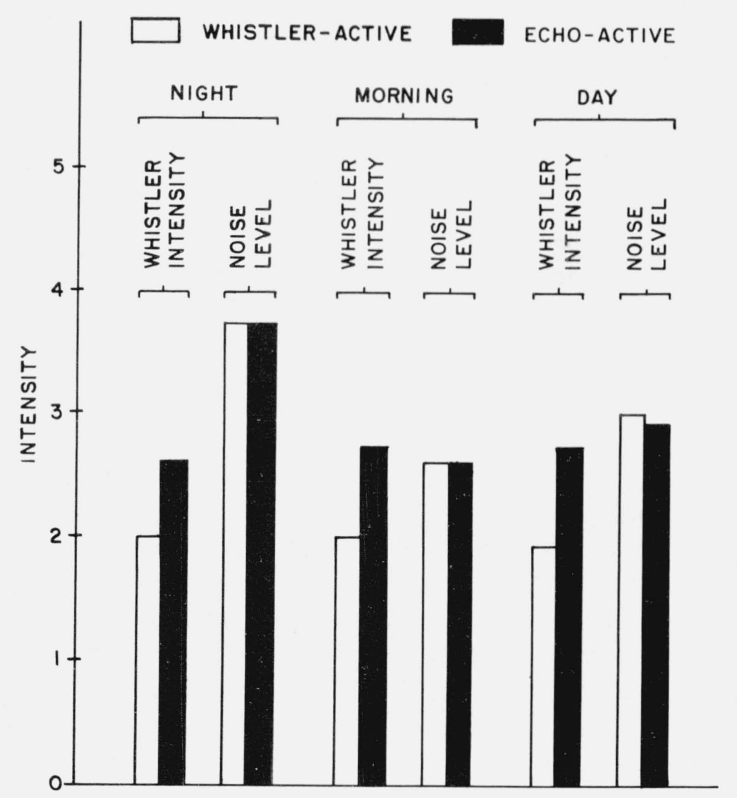

FIGURE 5. The intensity of the strongest whistler and the intensity of background noise (atmospherics) in whistler-active and echoactive periods at Dartmouth in the months of June and July, $1958-1963$.

A difference of unity on the intensity scale corresponds to about $10 \mathrm{~dB}$.

The results of figure 5 show that these higher rates are the result of increased whistler intensity, not of decreased atmospheric noise level. 
The reader is reminded that nearly all of Dartmouth's whistlers are long. A reflection mechanism must thus be operative in the-southern hemisphere not only during Dartmouth's echo-active periods but also during Dartmouth's whistler-active periods. For this reason we may assume that during both sets of observing periods the ionospheric conditions in the southern hemisphere are the same. This explains why the whistler rates in the southern hemisphere remain unchanged.

We conclude from the results that when (long) whistlers are observed at our local station, the question of whether or not these whistlers have echoes is determined by conditions existing at the receiver end of the path. The result that the "echoing situation" is characterized by whistlers of higher intensity is something our simple ionospheric trapping theory does not predict (see table 1). From the observation that the average noise level during echo-active and whistleractive periods is the same, we conclude that the echo occurrence is more an "on" or "off" situation than simply a result of small changes in the signal to noise ratio. This, by the way, also applies to the occurrence of long whistlers at Dartmouth: at local noon they are usually "off", and as shown by curves given by Laaspere, Morgan, and Johnson [1963], a deep depression in the average whistler rate results if all observing periods are used in the computations. As shown by the results quoted in section 3 , however, if those observing periods are discounted in which no whistlers were observed, the noontime and midnight rates are practically the same.

\subsection{Echo Occurrence in Conjugate Areas}

A comparison of the records of conjugate-area stations shows that an intense whistler echo train observed in one hemisphere is usually detected in the other hemisphere as well, at least as a single whistler. However, it is not uncommon to observe long whistlers in the northern hemisphere or short whistlers with echoes in the southern hemisphere which are not detected in the other hemisphere. Since the station coverage in our network is not complete and the observing conditions at the stations differ, it is not clear how many of these cases actually correspond to total reflection of the echo trains in the ionosphere. For a study of this kind it is best to have a relatively dense network of whistler stations in at least one hemisphere. We hope to have such a coverage soon at our network with six or seven stations in operation in the northern hemisphere.

Time periods exist when the echo occurrence is extremely high, but we have not yet been able to determine the cause of this. An exceptionally favorable echoing situation prevailed at our stations during many of the observing periods in the second half of December 1960, but even then the observations were highly variable. An echo train which occurred on December 22, 1960 at $07 \mathrm{hr} 50 \mathrm{~min} 45 \mathrm{sec}$ UT was observed at all of the Whistlers-East stations then in operation (Moisie, Quebec; Dartmouth College; Ber- muda; Port Lockroy and Ellsworth). On the other hand, some echo trains observed at our local station went undetected at all of the other stations.

\section{Echoes and Geomagnetic Activity}

It is of interest to point out that of the seven universal-time days listed in tables 2 and 3 , several follow geomagnetic storms by about a day. This raises the question of a possible association of whistler echoes with geomagnetic disturbances.

To check on this possibility, we first made an analysis of the stormtime variation of echo activity in more than 50 isolated geomagnetic storms, i.e., storms which were preceded and followed by a period of relatively quiet conditions. The results show that in the three days preceding the sudden commencement of the storms, an average of 18 percent of Dartmouth's whistler-active observing periods had echoes, compared to an average of 20 percent in the four days after the sudden commencement. The same analysis with the time of the peak disturbance (highest value of the $K_{p}$-index) as the reference shows that in the four days preceding the peak disturbance 18 percent of whistler-active periods had echoes, compared to 22 percent in the four days following the peak disturbance.

We have also determined the $K_{p}$-dependence of the probability of a whistler having an echo (percentage of whistlers with one or more echoes) at Dartmouth in the years 1957-1963. The result is plotted in figure 6 together with the $K_{p}$-dependence of the whistler rate. The practically identical shape of the two curves of figure 6 indicates that those $K_{p}$-dependent conditions which yield a high (or low) whistler rate also

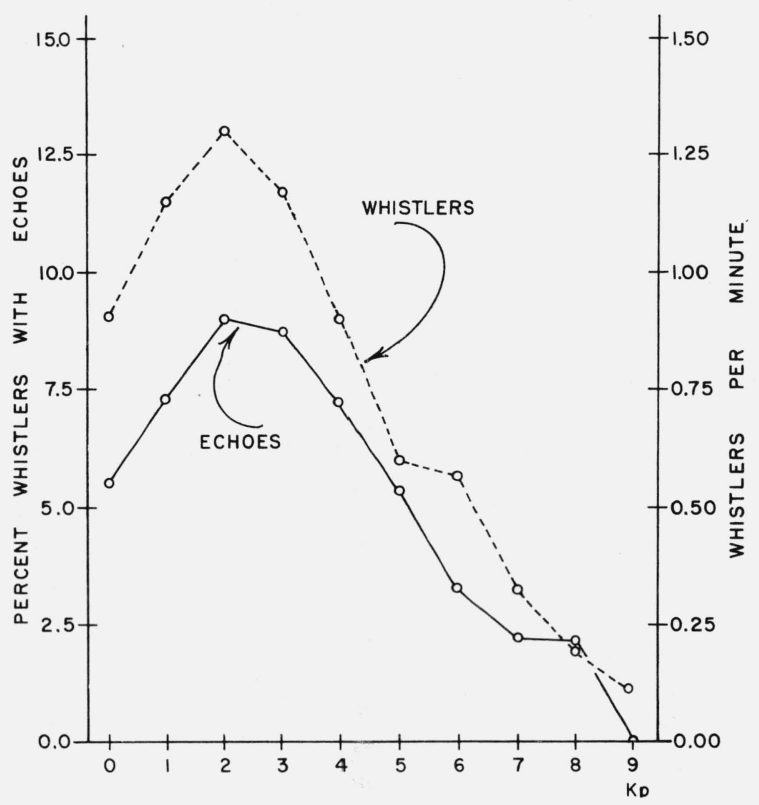

FIGURE 6. The $\mathrm{K}_{\mathrm{p}}$-dependence of the whistler rate and of the probability of a whistler having an echo at Dartmouth College, 19571963. 
enhance (or decrease) the likelihood of a whistler having an echo. Figure 6 shows that both the whistler rate and the echo occurrence are highest for $K_{p}$ around 2 or 3 , i.e., under relatively quiet geomagnetic conditions.

Figure 6 and the results on the stormtime variation of echo activity show clearly that most whistler echoes at Dartmouth have no association with geomagnetic storms. This does not, however, completely rule out the possibility that shortly after some geomagnetic disturbances conditions are especially good for observing intense whistler echo trains with perhaps unusually low intensity decrements. This is a point we are planning to analyze further.

It should also be pointed out that most of the whistlers listed in tables 2 and 3 were accompanied by natural hiss. It is, in fact, common to observe some hiss when echo activity is high. We do not believe that the presence of hiss in echo-active observing periods is an indication that an amplification mechanism of whistlers is operative, but that the hiss itself is often caused by whistler echo trains.

\section{Conclusion}

Our results indicate that whistler echo trains cannot be reflected at the ground or at the lower boundary of the daytime $D$ region unless the ionospheric transmission loss for whistler-mode waves at kilocycle frequencies for a one-way passage through the ionosphere is sometimes as small as $3 \mathrm{~dB}$ even at midday.

The whistler echo results could be explained by a magnetospheric trapping mechanism in which a whistler may be pictured bouncing back and forth between the ionospheres of the two opposite hemispheres, with some of the energy "leaking through" to the ground at one or both ends of the path. The exact nature of the "echo situation" is not clear, however, and more must be involved than simple reflection at electron density gradients.

At our local station the occurrence of long whistlers can be characterized reasonably well by the statement that the phenomenon is either "on" or "off". This description applies even better to the occurrence of the echoes of these whistlers. Insofar as conditions existing at the southern-hemisphere end of the path are concerned, the requirements for the occurrence at Dartmouth of long whistlers and whistler echoes appear to be the same. In the hemisphere of the receiver, however, the ionospheric conditions must apparently satisfy some additional requirements for the long whistlers also to have echoes.

The results of this paper point out the need for further studies of the propagation of whistler-mode waves in the ionosphere. Special attention should be given to the conditions leading to ionospheric reflection and trapping of these waves. It would be equally important to determine the ionospheric transmission loss for a wave already propagating in the whistler mode (such as downcoming chorus, hiss, etc.) from simultaneous measurements made in the ionosphere and at the ground. Further studies of whistler echoes also appear very promising and are being continued.

\section{Appendix}

We wish to determine here the intensity decrements in a whistler echo train for the different propagation models.

Let

$\rho_{i j}=$ power reflection coefficient of the "whistlermode" wave at the interface of regions $i$ and $j$ with the wave incident from the direction $i$;

$R_{i}=$ ratio of the intensity of the wave after having traversed region $i$ to the intensity of the wave when it entered the region $\left(R_{i}\right.$ will be called the "absorption factor");

$P_{i j}=$ intensity of the whistler incident at the interface of regions $i$ and $j$ from the direction of region $i$;

$P_{i j}^{(n)}=$ intensity of the $n$th whistler echo incident at the interface of regions $i$ and $j$ from the direction of region $i$.

\subsection{Intensity Decrements in the Ground Reflection Model}

We will first calculate the intensity decrement between a whistler and its echo for the ground reflection model. For purposes of generality we will include the partially reflecting surfaces even in this case, although the only effect of partial reflections in this model is to reduce the intensity of the whistler and its echoes.

Using the definitions given previously, the intensity of a downcoming whistler incident at the ground surface 2-1 is $P_{21}$, giving rise to a reflected wave of intensity $\rho_{21} P_{21}$. After absorption in region 2 , the whistler arrives at the interface $2-3$ with intensity $R_{2} \rho_{21} P_{21}$. Writing the power transmission coefficients in the form $1-\rho_{i j}$, the intensity of the whistler transmitted into region 3 is $\left(1-\rho_{23}\right) R_{2} \rho_{21} P_{21}$, the intensity of the wave at the interface $3-4$ is $R_{3}\left(1-\rho_{23}\right) R_{2} \rho_{21} P_{21}$, etc. Following this simple procedure, we obtain for the ratio of the intensity of the whistler echo to that of the whistler the following expression (more generally, this is also the ratio of intensities of the $n$th and $(n-1)$ th echoes):

$$
\begin{aligned}
& \frac{P_{21}^{(1)}}{P_{21}}=\frac{P_{21}^{(n)}}{P_{21}^{(n-1)}}=R_{2}^{2} R_{3}^{2} R_{4}^{2} \rho_{21} \rho_{45}\left(1-\rho_{23}\right) \\
& \quad\left(1-\rho_{34}\right)\left(1-\rho_{43}\right)\left(1-\rho_{32}\right) .
\end{aligned}
$$

In the pure ground reflection model the partially reflecting interfaces $2-3$ and $3-4$ are absent, and we have

$$
\frac{P_{21}^{(1)}}{P_{21}}=\frac{P_{21}^{(n)}}{P_{21}^{(n-1)}}=R_{2}^{2} R_{3}^{2} R_{4}^{2} \rho_{21} \rho_{45}
$$

The decibel intensity decrements in a whistler echo train in the ground reflection model are thus given by the expression

$-20 \log R_{2}-20 \log R_{3}-20 \log R_{4}-10 \log \rho_{21}$

$$
-10 \log \rho_{45} \text {. }
$$


The minus sign is introduced to make the intensity decrement a positive number.

\subsection{Intensity Decrements in the Trapping Model}

Let the intensity of a whistler incident from above on the partially reflecting surface or interface 3-2 be $P_{32}$. This gives rise to a "leakage whistler" of intensity $R_{2}\left(1-\rho_{32}\right) P_{32}$ at the ground surface $2-1$.

To determine the intensity of the leakage wave at $2-1$ at the second bounce of the wave, i.e., the intensity of the "echo", note that the original whistler incident from above on 3-2 gave rise to a reflected wave of intensity $\rho_{32} P_{32}$. This wave arrived at the interface 3-4 with the intensity $R_{3} \rho_{32} P_{32}$, was reflected back with intensity $\rho_{34} R_{3} \rho_{32} P_{32}$, etc. The intensity of the "echo" at $2-1$ is easily shown to be

$$
\mathrm{P}_{21}^{(1)}=\rho_{32} \rho_{34} R_{2} R_{3}^{2}\left(1-\rho_{32}\right) P_{32} .
$$

Since $R_{2}\left(1-\rho_{32}\right) P_{32}$ was the intensity of the "leakage whistler", we obtain for the ratio of the intensity of the "echo" to that of the whistler in the trapping model the following expression (this is also the ratio of intensities of the $n$th and $(n-1)$ th echoes):

$$
\frac{P_{21}^{(1)}}{P_{21}}=\frac{P_{21}^{(n)}}{P_{21}^{(n-1)}}=\rho_{32} \rho_{34} R_{3}^{2} .
$$

\subsection{Intensity Decrements in Mixed Models}

Using the procedure outlined previously, it is easy to determine the ratio of the intensity of the whistler echo to that of the whistler if a partially reflecting surface is present only in one hemisphere. In particular, if in figure 1 observations are made at the ground surface $2-1$ and the interface $3-4$ is removed, we obtain

$$
\frac{P_{21}^{(1)}}{P_{21}}=\frac{P_{21}^{(n)}}{P_{21}^{(n-1)}}=R_{3}^{2} R_{4}^{2} \rho_{32} \rho_{45} .
$$

If, on the other hand, the interface $2-3$ is removed (this is the interface in the hemisphere of the observer), we obtain

$$
\frac{P_{21}^{(1)}}{P_{21}}=\frac{P_{21}^{(n)}}{P_{21}^{(n-1)}}=R_{2}^{2} R_{3}^{2} \rho_{21} \rho_{34}
$$

The whistler stations at which these observations were made is part of a chain which was set up under the supervision of M. G. Morgan as part of the United States Program for the International Geophysical Year. The whole program was supported initially by the USA National Committee for the IGY and subsequently by successive grants from the National Science Foundation. The current work is supported by the Atmospheric Sciences Section of the National Science Foundation under Grant NSF GP-919.
We wish to acknowledge the conscientious efforts of W. Pacheco, a student from Bolivia, who determined the intensity decrements listed in tables 2 and 3. We also wish to acknowledge the contribution of our data analyst, Donald P. Harlow.

\section{References}

Altman, C., and H. Cory (1962), The transmission of audio-frequency electromagnetic waves through the terrestrial ionosphere in the magnetoionic mode, J. Geophys. Res. 67, 4086-4090.

Cartwright, D. G. (1964), Rocket observations of very low frequency radio noise at night, Planetary Space Sci. 12, 11-16.

Eckersley, T. L. (1928), Letter to the editor, Nature 122, 768.

Gallet, R. M. (1963), Whistlers, a chapter in Geophysics, The Earth's Environment ed. C. DeWitt, J. Hieblot, and A. Lebeau (Gordon and Breach, New York).

Helliwell, R. A. (1963), Coupling between the ionosphere and the earth-ionosphere waveguide at very low frequencies, Proc. International Conference on the Ionosphere, 452-460. (Inst. of Physics and the Physical Soc., London.)

Helliwell, R. A., and M. G. Morgan (1959), Atmospheric whistlers, Proc. IRE 47, 200-208.

Helliwell, R. A., and D. L. Carpenter (March, 1961), Whistlers-West IGY-IGC Synoptic Program, Final Report, NSF Grants IGY 6.10/20 and G-8839, Radiosci. Lab., St anford Univ.

Helliwell, R. A., J. Katsufrakis, and G. Carpenter (March, 1962), Whistler-mode propagation studies using Navy VLF transmitters, Techn. Report 1, ONR Contract Nonr 255 (27), Radiosci. Lab., Stanford Univ.

Hodara, H. (1962), Some remarks on 'penetration of the ionosphere by very-low-frequency signals,' Proc. IRE 50, 2000.

Jesperson, J. L., and M. L. V. Pitteway (December, 1963), The effect of the lower ionosphere on signals propagating in the whistler mode, Paper delivered at the URSI Fall Meeting, Seattle, Washington.

Laaspere, T., M. G. Morgan, and W. C. Johnson (1963), Some results of five years of whistler observations from Labrador to Antarctica, Proc. IEEE 5 1, 554-568.

Leiphart, J. P., R. W. Zeek, L. S. Bearce, and E. Toth (1962), Penetration of the ionosphere by very-low-frequency radio signals interim results of the LOFTI I experiment, Proc. IRE 50, 6-17.

Lomax, J. B. (June 1961), Measurement of VLF-transmission characteristics of the ionosphere with instrumented Nike-Cajun rockets, Final Report, Contract NOw 60-0405 (FBM), Stanford Res. Inst.

Morgan, M. G., and W. C. Johnson (May 1960), Whistlers along W $65^{\circ}$ longitude ('Whistlers-East') during the IGY and IGC 1959. Paper delivered at the URSI Spring Meeting, Washington, D.C.

Smith, R. L. (1961), Propagation characteristics of whistlers trapped in field-aligned columns of enhanced ionization, J. Geophys. Res. 66, No. 11, 3699-3707.

Storey, L. R. O. (1953), An investigation of whistling atmospherics, Phil. Trans. Roy. Soc. (London) A246, 113-141.

Swift, D. W. (1962), Very-low-frequency radio propagation in the ionosphere, J. Res. NBS 66D (Radio Prop.), No. 6, 663-680.

Watt, A. D., and E. L. Maxwell (1957), Characteristics of atmospheric noise from 1 to $100 \mathrm{kc}$, Proc. IRE 45, 787-794.

\section{Additional Related Reference}

Vestine, E. H., and W. L. Sibley (1960), The geomagnetic field in space, ring currents and auroral isochasma, J. Geophys. Res. 65, 1967-1979. 